\title{
X-Ray Spectroscopy of Astrophysically- Relevant Photoionized Iron Plasmas at Z
}

\author{
R.F. Heeter, J.E. Bailey, M.E. Cuneo, J. Emig \\ M.E. Foord, P.T. Springer, R.S. Thoe
}

This article was submitted to $12^{\text {th }}$ APS Topical Conference on Atomic Processes in Plasmas Reno, NV, March 19-23, 2000

\section{June 12, 2000}

U.S. Department of Energy

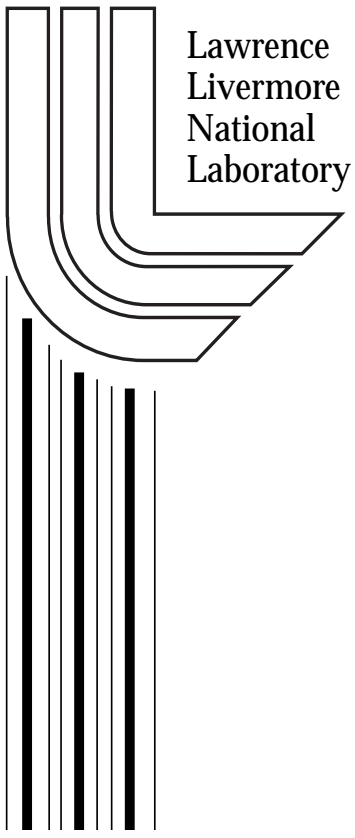




\section{DISCLAIMER}

This document was prepared as an account of work sponsored by an agency of the United States Government. Neither the United States Government nor the University of California nor any of their employees, makes any warranty, express or implied, or assumes any legal liability or responsibility for the accuracy, completeness, or usefulness of any information, apparatus, product, or process disclosed, or represents that its use would not infringe privately owned rights. Reference herein to any specific commercial product, process, or service by trade name, trademark, manufacturer, or otherwise, does not necessarily constitute or imply its endorsement, recommendation, or favoring by the United States Government or the University of California. The views and opinions of authors expressed herein do not necessarily state or reflect those of the United States Government or the University of California, and shall not be used for advertising or product endorsement purposes.

This is a preprint of a paper intended for publication in a journal or proceedings. Since changes may be made before publication, this preprint is made available with the understanding that it will not be cited or reproduced without the permission of the author.

This report has been reproduced directly from the best available copy.

Available to DOE and DOE contractors from the

Office of Scientific and Technical Information

P.O. Box 62, Oak Ridge, TN 37831

Prices available from (423) 576-8401

http:/ / apollo.osti.gov/bridge/

Available to the public from the National Technical Information Service

U.S. Department of Commerce 5285 Port Royal Rd., Springfield, VA 22161 http://www.ntis.gov/

OR

Lawrence Livermore National Laboratory Technical Information Department's Digital Library http://www.llnl.gov/tid/Library.html 


\title{
X-Ray Spectroscopy of Astrophysically-Relevant Photoionized Iron Plasmas at Z
}

\author{
R. F. Heeter*, J. E. Bailey ${ }^{\dagger}$, M. E. Cuneo ${ }^{\dagger}$, J. Emig*, M. E. Foord*, \\ P. T. Springer*, and R. S. Thoe* \\ ${ }^{*}$ Lawrence Livermore National Laboratory ${ }^{1}$ P. O. Box 808, Livermore CA 94550 \\ ${ }^{\dagger}$ Sandia National Laboratories, P. O. Box 5800, Albuquerque, NM 87185
}

\begin{abstract}
In order to provide benchmark data for models used to interpret X-ray astronomy data from newly-launched orbital telescopes such as Chandra, we have used $120 \mathrm{TW}, 180 \mathrm{eV}$ pinch plasmas on the Sandia Z facility to drive iron foils into X-ray photoionized equilibrium. The experiment was designed to achieve photoionization parameters characteristic of accretion-powered objects such as X-ray binaries (neutron stars) and active galactic nuclei (black holes). These objects comprise roughly half of observed X-ray sources, but the interpretation of their spectra is difficult: stateof-the-art models for photoionized iron plasmas do not yet agree on the expected ionization balance. In our initial experiments the foil samples consisted of $200 \AA$ of iron codeposited with $300 \AA$ of sodium fluoride and sandwiched between two $1000 \AA$ layers of Lexan $(\mathrm{CH}$ and $\mathrm{O})$. We characterized the pinch spectrum, temperature, power and uniformity and qualified it as a photoionization driver. We obtained time-integrated absorption spectra for the foil from 8 to $18 \AA$ and identified spectral lines from $\mathrm{O}$ VIII, F IX, Na X and XI, and Fe XVII and XVIII, i.e. neon-line and fluorine-like iron. Time-resolved absorption and emission spectra for the foils were also obtained from 12.5 to $16 \AA$, and hydrogen-like $\mathrm{F}$ and neon-like and fluorine-like Fe were again observed in the $2 \mathrm{~ns}$ time window of interest. In subsequent ridealong experiments we have developed a density diagnostic and measured the density via foil-expansion imaging at two locations. We conclude by discussing upcoming experiments at $\mathrm{Z}$ in which we plan to obtain a full data set of plasma density, temperature, and absorption and emisssion spectra for multiple photoionization equilibria.
\end{abstract}

\section{INTRODUCTION}

This paper presents the basic approach and first results of laboratory experiments developed to help resolve one of the important physics challenges arising in contemporary X-ray astronomy, and outlines the progress which is expected in this

1) This work was performed under the auspices of the U.S. Department of Energy by University of California Lawrence Livermore National Laboratory under contract no. W-7405-Eng-48. 
area in the near term. A large fraction of astrophysical X-ray sources are accretionpowered objects where an intense core X-ray source illuminates and photoionizes the surrounding gas. The Chandra and XMM observatories are capable of obtaining spectroscopic data with much higher resolution than previously possible in $\mathrm{X}$-ray astronomy. However, the usefulness of excellent spectroscopic data depends greatly upon one's confidence in the basic atomic physics of the models with which the data is interpreted. Users of these data interpretation tools ought to be able to specify a set of plasma conditions, calculate the ionization balance and other properties of such a plasma, and get similar results from multiple codes. We present the results of such a comparison for five major photoionization equilibrium models in the next section. Confidence in the models can be greatly increased if the model predictions are directly benchmarked against experimental data obtained in well diagnosed laboratory experiments under known plasma conditions; however, until recently the photoionization dominated equilibrium was inaccessible to laboratory experiments. In subsequent we describe how Z-pinch driven intense X-ray sources now enable laboratory astrophysics experiments in the photoionization regime. Finally, we report the first results and future plans from such a project at the end of this paper. In future publications we expect to present fully diagnosed experimental photoionization data with which to better benchmark the models and thereby improve the physics foundations for X-ray astronomy.

\section{COMPARISON OF MODELS FOR PHOTOIONIZED PLASMAS}

The photoionization equilibrium in plasma physics arises when the dominant terms in the ionization balance equations are the processes of photoionization (absorbed photon ejects electron from ion) and recombination (electron recombines with ion and emits photon or excites another electron). This situation occurs when the ratio of the X-ray flux to the electron density is sufficiently high that collisional ionization processes are negligible relative to photoionization processes. We are particularly interested in the low density regime where 3-body recombination may also be neglected relative to 2-body recombination processes, such that

$$
n_{i} \Gamma_{\text {rad }} \int_{x}^{\infty} S(E) \sigma_{i}(E) E^{-1} d E=n_{e} n_{i+1} \alpha_{i+1} .
$$

On the left side, $n_{i}$ is the density of a given ion species, $\Gamma_{r a d}$ is the total radiation flux, and the integral term uses the spectral shape $S(E)$ and the photoionization cross section $\sigma_{i}(E)$ to determine the photoionization rate coefficient by integrating all energies above the relevant ionization threshold. On the right side, $n_{e}$ is the electron density, $n_{i+1}$ is the density of the next ionization stage, and $\alpha_{i+1}$ is the recombination rate coefficient for that stage. One traditionally sets $\xi \equiv \frac{4 \pi \Gamma_{\text {rad }}}{n_{e}}$ and it then follows from Equation 1 that the ionization balance $\frac{n_{i+1}}{n_{i}}$, and indeed much of the physics of these non-LTE photoionized plasmas, is determined by fundamental 
atomic physics data $(\sigma, \alpha)$, the spectral shape $S(E)$, and the photoionization parameter $\xi[1,2]$. It must be noted that these simple labels conceal a vast number of processes taking place at the atomic level, and that accounting for these processes accurately is a considerable computational challenge.

In order to assess which experiments might best benchmark the models used for photoionized plasmas, we obtained iron ionization balance predictions from five photoionization codes used in the astrophysical community at $\xi \approx 100 \mathrm{erg} \mathrm{cm} / \mathrm{s}$. The results appear in Figure 1, and show disagreement: predictions for the dominant charge state range from Fe XIII $(+12)$ to Fe XXIII $(+22)$. The comparison was done somewhat informally, but did specifically consider pure iron plasmas in the low density limit where 3-body processes were neglected. The divergence in the results is far greater than can be accounted for by the small range of photoionization parameters used. We obtained similar results in an earlier comparison of four of these five codes at $\xi \approx 150$ [3]. Although the physics of pure iron plasmas is somewhat different from plasmas with astrophysical abundances, models intended for complex plasmas with many elements ought to be accurate for single elements. These results should be considered preliminary, and consequently the codes are not individually identified until we can formalize the comparison. However, it appears that at present, users of these data analysis tools should expect considerable disagreement between them (either due to "pilot error" or differences in the physics) and that even simple ionization balance data would be useful in testing the models experimentally. We are actively collaborating with several photoionization modeling groups in this area, including the developers of CLOUDY [4], XSTAR [5,6] and ION (Netzer).

\section{Z PINCH X-RAY SOURCES FOR PHOTOIONIZATION}

The production of strongly X-ray photoionized plasmas in the laboratory has only recently become possible, with the advent of intense Z-pinch X-ray sources. To achieve a given photoionization parameter $\xi$, one needs to maximize the X-ray flux on a given sample while minimizing the sample density. The first consideration implies placing the sample as close as possible to the strongest possible X-ray source. The second consideration must be balanced against the need to obtain high quality absorption and emission signals, which require maximizing the product of density and scale length. To minimize the density one would like the largest possible sample, but the scale length is constrained by the characteristics of the X-ray source and the need to maintain the entire sample at roughly the same photoionization equilibrium.

The strongest laboratory X-ray source on Earth today is the Z Machine at Sandia National Laboratory, which routinely produces upwards of $100 \mathrm{TW}\left(10^{21} \mathrm{ergs} / \mathrm{s}\right)$ by driving $18 \mathrm{MA}$ of electrical current through cylindrical arrays of 100-400 fine wires $[7,8]$. The array as a whole is some $20-70 \mathrm{~mm}$ in diameter and $10-20 \mathrm{~mm}$ tall to start with. Each wire is on the order of 10 microns in diameter, about a tenth the thickness of a human hair. The Lorentz force acting on the parallel 


\section{Charge state distributions from astrophysical models}

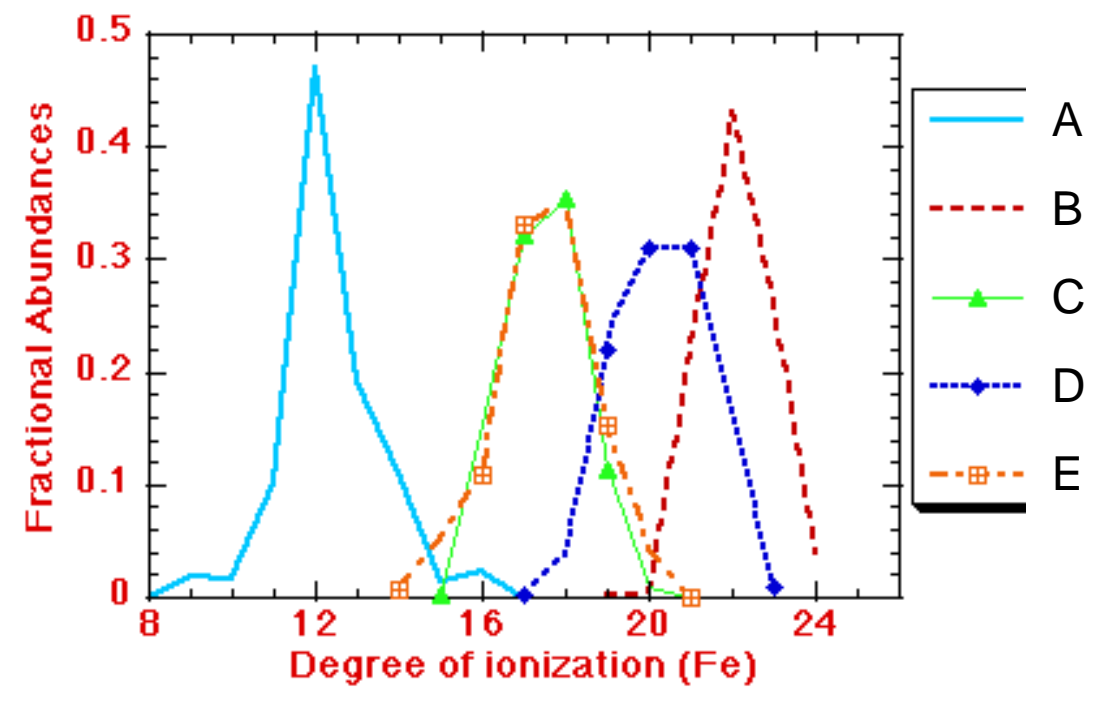

FIGURE 1. Informal comparison of five photoionization codes at $\xi \approx 100 \mathrm{erg} \mathrm{cm} / \mathrm{s}$.

currents causes the wire array to accelerate inwards, reaching velocities on the order of $100 \mathrm{~km} / \mathrm{s}$ over some $100 \mathrm{~ns}$. The convergence of the array results in stagnation on axis, at which time the kinetic energy of the imploding wires is converted into a 5-10 ns pulse of thermal X-rays with a characteristic temperature of 100-200 eV (1-2 million $\mathrm{K})$ from a region with a typical diameter of 1-2 $\mathrm{mm}$.

It is interesting to compare the $\mathrm{X}$-ray fluxes achieved at $\mathrm{Z}$ with astrophysical sources. Foil samples may be placed as close as 15 or $16 \mathrm{~mm}$ from the center of the pinch. Working in cylindrical geometry one finds that a $100 \mathrm{TW}, 1 \mathrm{~cm}$ long pinch produces an X-ray flux at the sample in excess of $3 \times 10^{19} \mathrm{ergs} / \mathrm{s} / \mathrm{cm}^{2}$, which is comparable to the X-ray flux just $1500 \mathrm{~km}$ from a neutron star with a luminosity of $10^{37} \mathrm{ergs} / \mathrm{s}$. The $100 \mathrm{~ns}$ run-in time of the pinch provides time for a foil to "blow down" to $10^{-4}$ or less of solid density, and enables Z to produce photoionization regimes with $\xi \sim 100 \mathrm{erg} \mathrm{cm} / \mathrm{s}$, comparable to conditions found in such accretion powered astrophysical objects as X-ray binaries and active galactic nuclei. Some care must be taken with this comparison because the details of the photoionizing spectrum, plasma composition and plasma density are necessarily different. However, all of these can be measured in the laboratory and accounted for in the modeling, and the fundamental physical processes governing the photoionization equilibrium remain the same, although some processes may be more important than others in the various cases. We therefore anticipate that benchmarking of the photoionization codes with Z-pinch data will enable astronomers to more accurately (or at least more confidently) use these models to interpret X-ray astronomy data. 


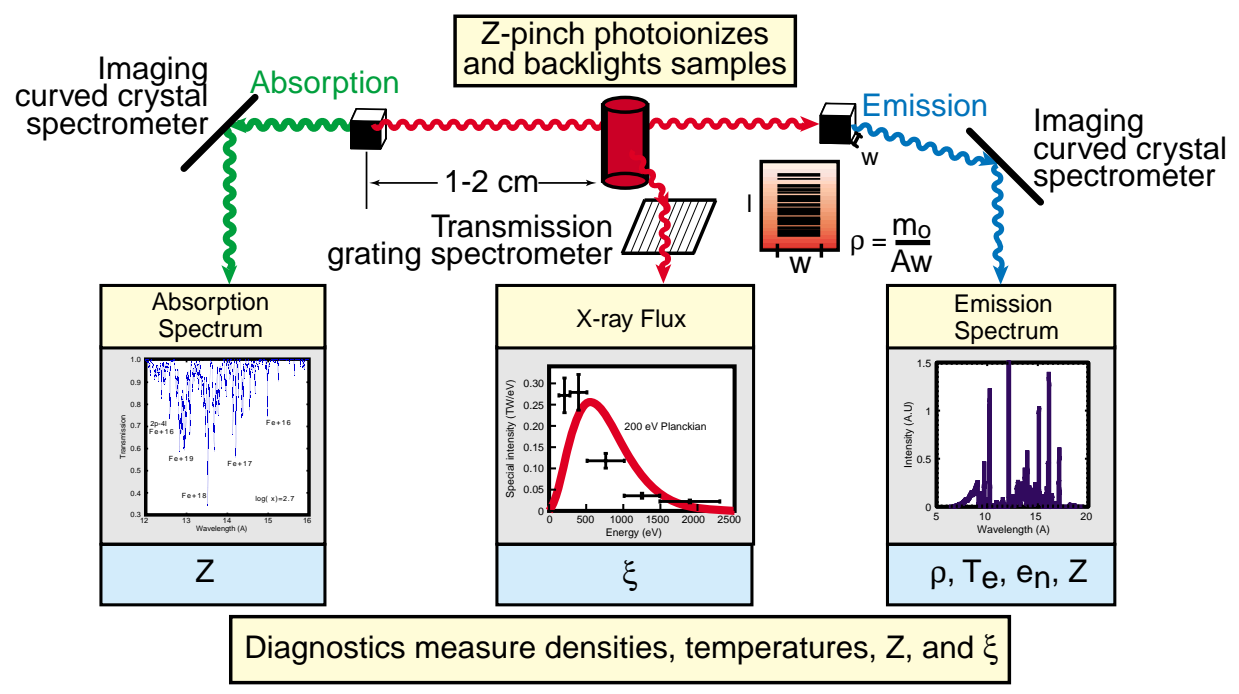

FIGURE 2. Experimental Concept.

\section{EXPERIMENTAL OBJECTIVES AND CONCEPT}

To properly benchmark the models, one must characterize the flux, spectrum (temperature) and uniformity of the pinch, photoionize a sample into an astrophysically interesting regime, and then measure the absorption and emission of the sample while simultaneously determining the sample density and temperature, preferably in a model independent way. The conceptual layout of such an experiment is shown in Figure 2. In this concept, the pinch is to be characterized using several Sandia diagnostics, which include time gated pinhole cameras, time resolved absolutely calibrated transmission grating spectrometers, calibrated X-ray diodes and bolometers, and a radially and temporally resolved X-ray streak camera. The sample absorption and emission, as well as one band of the pinch spectrum, are to be measured with one or more curved crystal spectrometers, with the Z pinch used as a backlighter for the absorption measurement. The ratio of the absorption spectrum to the pinch spectrum then yields the opacity. The sample temperature is to be extracted from the slope of the recombination continuum as well as line ratios of low-Z tracers in the sample. The sample density is to be obtained by imaging the expansion of the foil with a time gated pinhole camera or imaging spectrometer and comparing the final size with the known initial size and density of the foil.

Although reasonably straightforward in principle, this concept has a number of subtleties which require significant attention to be paid to the experimental design. For instance, in order to compare the time dependent experiment with time independent equilibrium models, the X-ray drive pulse must allow the sample enough time to come into equilibrium with the radiation field of the pinch. One must then produce time gated measurements of the sample in this "steady state" regime, before the X-ray pulse from the pinch terminates. One would also like the sample to ionize and expand in a uniform manner, so that all parts of the sample are 
in the same equilibrium. One also needs the sample to be sufficiently opaque that an absorption spectrum may be obtained, and sufficiently massive that an emission spectrum may be obtained. Given the millimeter scale lengths available in a Z pinch, these considerations all tend towards higher density samples. However, this conflicts with the desire to minimize the sample density in order to eliminate 3-body processes and maximize the photoionization parameter. One must therefore take care in designing the experiment, to ensure that a balance may be found whereby all these objectives can be achieved simultaneously.

These experimental design issues have been addressed using 1-D and 2-D time dependent radiation hydrodynamics simulations with the LASNEX code [9]. The resulting sample is a foil composed of $200 \AA$ of iron (the astrophysically interesting component) co-mixed with $300 \AA$ of sodium fluoride (tracers to be used as temperature diagnostics). The foil is hydrodynamically tamped with $1000 \AA$ of Lexan $(\mathrm{C}, \mathrm{H}$, and $\mathrm{O})$ placed on either side, forming a sandwich configuration. The foil is 1 $\mathrm{mm}$ high and $0.5 \mathrm{~mm}$ wide, and placed face-on to the pinch to provide maximum uniformity of illumination. As shown in Figure 3, the run-in phase of the pinch allows the foil to blow down, and the 5-10 ns duration of the X-ray pulse is such that the foil conditions reach equilibrium from 2-4 ns following the peak of the $\mathrm{X}$-ray pulse. (Here equilibrium conditions are defined as conditions whose values at a given time match the values expected in steady state for the same X-ray drive at that time.) This 2 ns time window poses one of the key challenges for the experiment: it forces the use of time gated rather than time integrated spectrometers to obtain the actual photoionization data, and is comparable to the timing jitter of the best available trigger signals on the machine. At the start of this 2 ns window, we calculate that the iron part of the foil will have expanded (in thickness) from 0.5 to 1200 microns, with less than $10 \%$ variations in density and temperature across the foil. This maximizes $\xi$ by bringing the foil density down as much as possible, and also allows the expansion of the foil to be imaged edge-on with a time gated pinhole camera. Based on 2-D (R-Z) calculations which account for the actual pinch geometry, we expect an electron density $n_{e} \sim 10^{19} / \mathrm{cm}^{3}$ and a mass density $\rho \approx 10^{-4}$ to $10^{-5} \mathrm{~g} / \mathrm{cm}^{3}$, for $\xi$ on the order of 10 to $100 \mathrm{erg} \mathrm{cm} / \mathrm{s}$. Under these conditions, preliminary calculations suggest that the charge balance is insensitive to 3-body recombination effects and the low-density photoionization equilibrium described in Equation 1 is reached, although further modeling (with experimental data) is required to confirm this.

\section{INITIAL EXPERIMENTS AND RESULTS}

Two preliminary photoionization experiments were carried out at Z in September 1999. The principal objectives of these two "shots" were to characterize in detail a particular pinch configuration and to validate the basic experimental design by obtaining as much foil data as possible. We used a $20 \mathrm{~mm}$ diameter tungsten wire array with 300 wires, each $10 \mu \mathrm{m}$ in diameter. Tungsten was chosen because it 

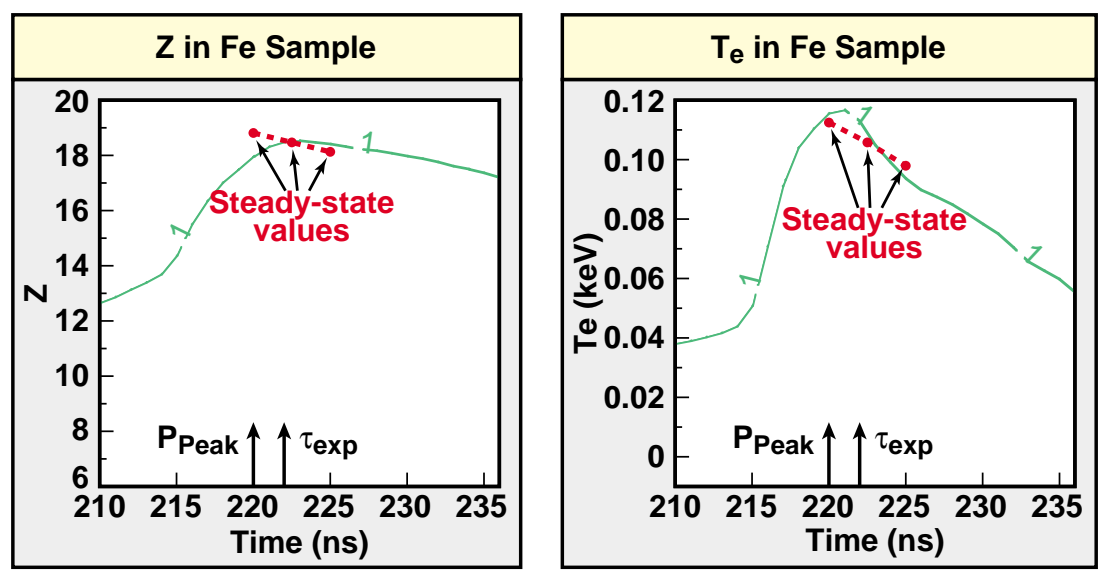

FIGURE 3. Simulated iron ionization $(\mathrm{Z})$ and electron temperature $\left(\mathrm{T}_{\mathrm{e}}\right)$ vs. time, with equivalent steady-state values shown.

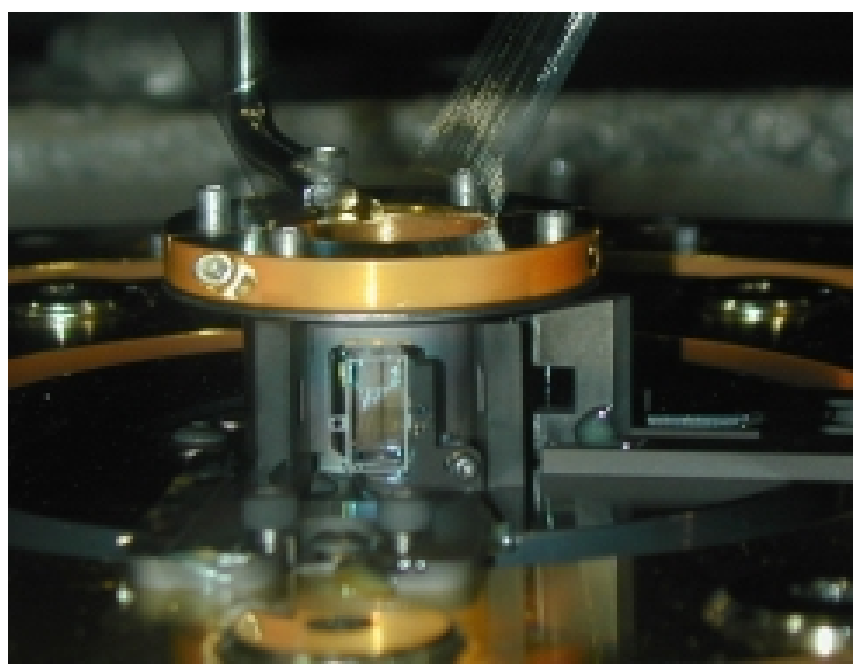

FIGURE 4. Photo of first generation experimental target.

has a line-free, thermal spectrum in the spectral range of interest $(750-1300 \mathrm{eV}$, or roughly $8-16 \AA$ ), and the small $(20 \mathrm{~mm})$ many-wire pinch was chosen to provide the maximum X-ray flux onto the sample.

Figure 4 provides a view of the actual first-generation "target" (experiment). The wires of the array can be seen diverging out the top of the current return can, which is basically a cylinder with slots cut out to allow instruments to see through to the pinch. Two of the experimental foils can be seen: the first is face-on to the camera in the center of the picture, and the second is seen edge-on off to the right. Various graphite windows were used to limit the fields of view of various instruments. Graphite was chosen so that in the spectral range of interest only the foils and pinch would be visible. For the same reason, all pinch-facing high-Z surfaces were coated first with gold and then with boron. A schematic top view of the experiment is shown in Figure 5, which also details the various diagnostic 


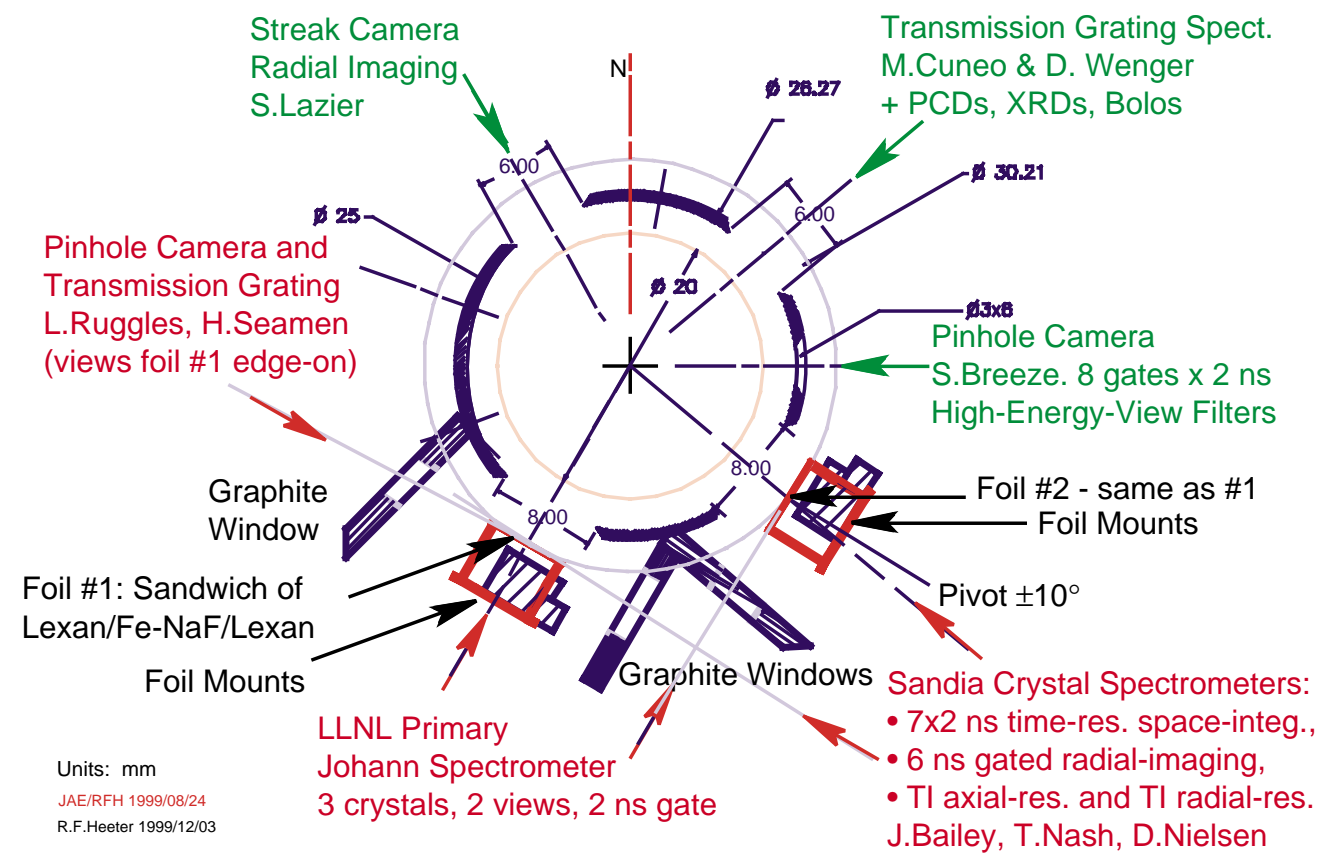

FIGURE 5. Schematic of first generation experiment, indicating relevant instrument lines of sight and responsible Sandia diagnostics personnel from the $\mathrm{Z}$ team.

instruments and their lines of sight. The view shown in Figure 4 is approximately the view seen by the LLNL Johann Spectrometer (lower left in Figure 5).

The results of these initial experiments are as follows. Reasonable success was achieved with the time-gated diagnostics; all diagnostics were gated across the time of interest (2-4 ns after the peak X-ray power) on at least one of the two shots. In both shots (Z476 and Z477), the pinch produced $120 \mathrm{TW}( \pm 10 \%)$ of predominantly thermal X-rays with a characteristic temperature of $180 \mathrm{eV} \mathrm{[10].} \mathrm{At} \mathrm{the} \mathrm{time} \mathrm{of}$ interest, radially and temporally resolved streak camera data gives a FWHM of $1.5 \mathrm{~mm}$ for the pinch diameter [11]. Pinhole camera images of the X-ray emission above roughly $600 \mathrm{eV}$ show adequate uniformity of the pinch at this time [12].

The experiments also measured the production of L-shell iron species in the foil samples. A crystal spectrometer with spatial resolution along the pinch axis (vertical) direction obtained a high quality time integrated absorption spectrum, shown in Figure 6, with tentative line identifications using a semi-empirical wavelength scale [13]. The upper trace is a lineout taken through the top part of the foil, where no iron was deposited, and is simply the pinch spectrum with absorption from the Lexan $(\mathrm{C}, \mathrm{H}, \mathrm{O})$ part of the foil. The lower trace is a lineout taken through part of the foil containing the Fe:NaF mixture, and shows numerous lines from the sodium Lyman and Helium series, fluorine Lyman series, and both neon-like and fluorinelike iron (Fe XVII and Fe XVIII). Of particular interest is the Fe XVII 2p-nd series, with $\mathrm{n}$ ranging from 3 to at least 8 . The iron spectral lines were identified based on recent data from the EBIT facility at LLNL $[14,15]$. We note that the relative line strengths in photoionized plasmas are expected to be considerably different from 


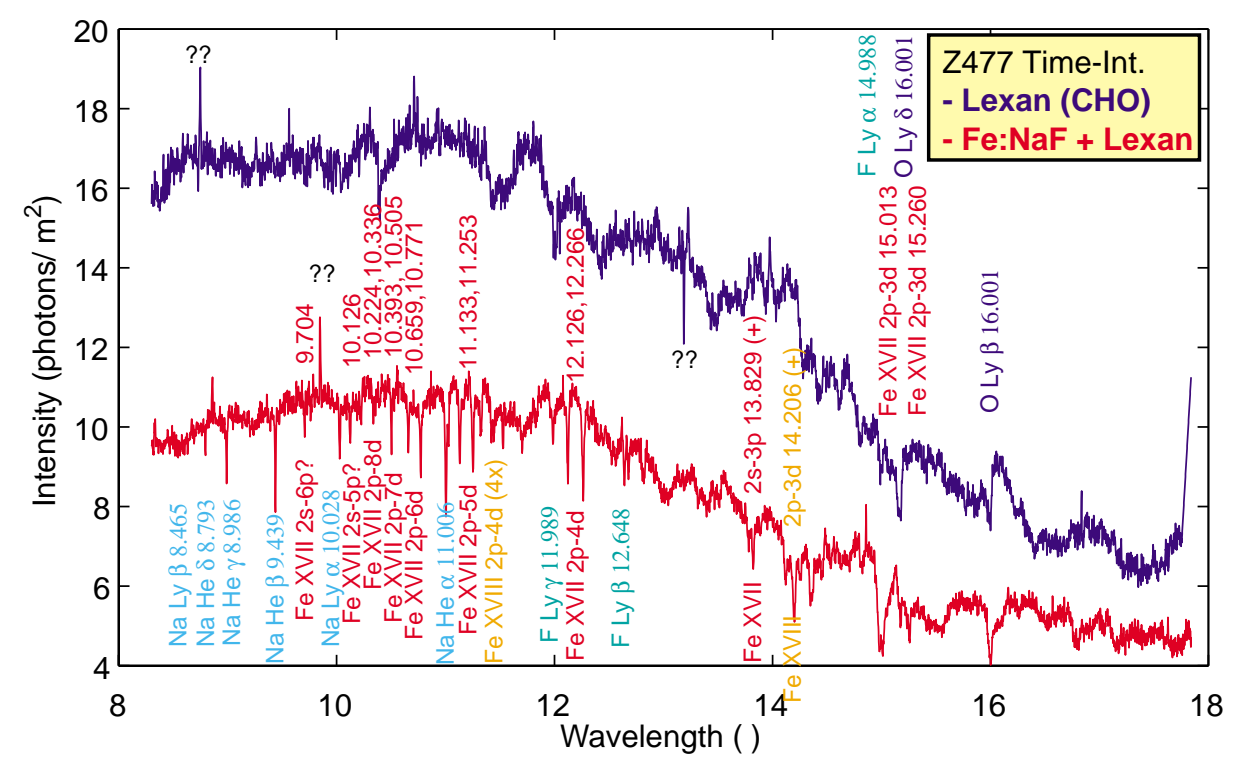

FIGURE 6. Time integrated spectra from a KAP convex crystal spectrometer.

those in collisional plasmas, which complicates the line identification process, especially when one has only one or two spectra to analyze [16]. The time integrated spectrum in Figure 6 is not ideal to benchmark photoionization models, since over much of the integration period the foil was not expected to be in the photoionized regime. However, the spectrum validates our ability to photoionize Fe into the $\mathrm{L}$ shell and produce highly ionized $\mathrm{Na}$ and $\mathrm{F}$ for spectral diagnostic use.

To obtain time resolved absorption and emission data we fielded a 3-crystal, 2-ns gated Johann curved crystal spectrometer belonging to LLNL. This spectrometer had not previously been used at Z, and a number of difficulties were encountered in this "shakedown" experiment, but we did obtain time gated data from 1-3 ns after the X-ray peak (a respectably close 1 ns from the desired "steady state" window), albeit with higher backgrounds than we would have liked. In these spectra we have also identified lines belonging to Ne-like Fe XVII in absorption, along with the Lyman- $\alpha$ line of Fluorine, plus Fe XVII and Fe XVIII in emission.

It was hoped that the sample density would be determined by imaging the expansion of the foil with a time-resolved pinhole camera viewing the foil edge-on through a graphite window. However, we found that the field of view was contaminated by boron blowing back from the wall of the can and by carbon blowing off the walls of the graphite windows (see Figure 4), and with only two shots we were unable to properly tune the instrument filtering and gain settings in order to properly resolve the small amount of iron against these background sources.

Perhaps the most important result of this initial experiment was in validating the basic experimental design and supplying critical data for subsequent designs. The initial design was made using a number of approximations for the pinch power and spectrum as a function of time, and as shown in Figure 1 there was considerable 
uncertainty about the expected ionization of iron, but the foil ionized roughly as predicted. Since we now have absolutely-calibrated data for the pinch power and spectrum, future designs should also work. We had some concern about unanticipated magneto-hydrodynamic effects altering the foil dynamics in ways which could not be accounted for in the modeling, but we have not seen any evidence of these so far. Finally, the specific pinch configuration which we used had not been tried before, so there was some concern that the asymmetry of the return current can might spoil the pinch implosion, but the actual shots were successful.

\section{CONCLUSIONS AND FUTURE WORK}

Photoionized plasmas are common in astrophysics, and detailed X-ray spectra of such plasmas are now being obtained by new missions such as Chandra and XMM. However, interpretation of these spectra rely on models which are difficult to bring into agreement. We have developed the first capability to produce nearsteady-state, uniform photoionized plasmas in the laboratory, using foil samples placed near the Sandia Z Machine. In our first generation experiments the pinch worked well as a photoionization driver. We have evidence for the production of photoionized L-shell iron, including both time integrated and time resolved spectra with Fe XVII and Fe XVIII seen in both emission and absorption.

Based on these promising initial results, at the time of this conference we had prepared and planned an improved second-generation experimental run to be carried out in April and May 2000. The three principal changes in the experiment improved the time-resolved spectral measurements, the time-resolved density measurement, and the plasma temperature measurement, respectively.

To improve the time-resolved spectroscopy of the photoionized samples, multiple improvements were made in the line of sight baffle, shielding, and alignment systems on the time-resolved dual-sightline Johann spectrometer. The main difficulties which these improvements addressed were the need to actively monitor the instrument alignment while under vacuum, the need to fully separate the light coming from the emission foil from the light coming from the much brighter pinch (and foil in absorption) only $1.5 \mathrm{~cm}$ away, and the need to better reject stray radiation.

To successfully measure the expansion of the foil (and get the foil density) we have taken two approaches. The first is to improve the pinhole camera measurements. We redesigned the pinhole camera filters to better discriminate the iron in the foil from other sources that might be visible, and we also modified the hardware along the camera's line of sight to reduce blowoff into the camera's field of view. The second approach is to redesign the foils to sit in a horizontal plane edge-on to the pinch. This allows the vertical foil expansion to be imaged in absorption with space and time resolved spectrometers, as well as in emission by the pinhole camera. To test the horizontal-foil approach, we did two ridealong experiments in which surplus foils from the first-generation experiment were positioned edgeon to a pinch and viewed in absorption using the time-integrated, space-resolved 
spectrometer discussed above. By determining the spatial extent of the oxygen and iron lines in these spectra, we have determined the expansion of the Lexan tamper and of the iron in the sample, as desired. To ensure that these horizontal foils would again expand uniformly without lingering shocks or unwanted temperature or density gradients, the foils were completely redesigned, again using LASNEX. The foil mounting hardware was also greatly simplified, to speed up the experimental setup and to prevent contamination of the line of sight with unwanted blowoff. In these second-generation foils, the iron and sodium fluoride layers have the same composition as before, but they are $8 \mathrm{~mm}$ long by $2 \mathrm{~mm}$ wide, and are suspended by a Lexan bridge across the slot in the current return can. The standard configuration uses the same Lexan thickness as before. The Lexan thickness can be varied to provide varying amounts of inertia, which allows the foil to expand to a different final thickness (density). This technique makes it possible to perform scans in the photoionization parameter, given enough experimental shots.

Finally, to improve the prospects for a plasma temperature measurement, we developed a heavily shielded time-integrated flat crystal spectrometer to be placed close-in to the pinch and used to measure the slope of the free-bound continuum emission from the foil at around 1.5 to $2.0 \mathrm{keV}$. The shielding consisted of a minimum of $25 \mathrm{~mm}$ of tungsten on all sides except the line of sight. The line of sight will point through the foil from the side, so that only the foil and the vacuum vessel wall can shine directly into the spectrometer, which should reduce fogging of the film from stray radiation.

Based on these improvements, we believe that the four shots in this secondgeneration experiment should enable us to obtain the first well-characterized data set for these photoionized plasmas. Subsequent work will be needed to begin the process of benchmarking the models with this data, and to extend the experiments to different elements $(Z)$ and multiple photoionization parameters $(\xi)$. Scans in $Z$ and $\xi$ will provide much more detailed benchmarks and also make it easier to identify and resolve any weaknesses that may be found in the models. In conclusion, we note that these are merely the first steps in the laboratory astrophysics of accretion-powered objects: over the longer term one can envision new X-ray sources and sample compositions which will allow increasingly direct simulations of actual accretion-disk plasmas to be produced in the laboratory.

The authors especially thank T. Dimwoodie at Sandia for timing and data acquisition support. We also thank C. Coverdale, S. Breeze, D. Jobe, S. Lazier, J. McGurn, T. Nash and the Z Machine operations team at Sandia for experimental support, and the LLNL LDRD program for financial support.

\section{REFERENCES}

1. C. B. Tarter, W. Tucker, and E. E. Salpeter, Astrophysical Journal 156, 943 (1969).

2. B. Tarter et al., Astrophysical Journal 156, 953 (1969).

3. R. F. Heeter et al., in Atomic Data Needs for X-Ray Astronomy, edited by M. A. 
Bautista, T. R. Kallman, and A. K. Pradhan (NASA, Goddard Space Flight Center, Greenbelt, MD, 2000), pp. 135-142.

4. G. J. Ferland et al., Proc. Ast. Soc. Pacific 110, 761 (1998).

5. T. Kallman et al., Astrophysical Journal 465, 994 (1996).

6. T. Kallman and R. McCray, Astrophysical Journal Supplement 50, 263 (1982).

7. C. Deeney et al., Phys. Rev. Lett. 81, 4883 (1998).

8. M. G. Haines et al., Phys. Plasmas 7, 1672 (2000).

9. G. B. Zimmermann and W. L. Kruer, Comments on Plasma Phys. and Cont. Fusion 2, 85 (1975).

10. M. E. Cuneo, Private Communication, 1999.

11. S. E. Lazier, Private Communication, 1999.

12. S. Breeze, Private Communication, 1999.

13. J. E. Bailey, Private Communication, 1999.

14. G. V. Brown et al., Astrophysical Journal 502, 1015 (1998).

15. G. V. Brown, Ph.D. thesis, Auburn Univ., 2000.

16. D. A. Liedahl, S. M. Kahn, A. L. Osterheld, and W. H. Goldstein, Astrophysical Journal 350, L37 (1990). 